\title{
An Unusual Case of Guava Seed Embedded in Buccal Mucosa for Past 6 Months
}

\author{
${ }^{1}$ Shruti Sharma, ${ }^{2}$ Dipika Bumb, ${ }^{3}$ Sumon Sen, ${ }^{4}$ Smita Priyadardshini
}

\begin{abstract}
Foreign bodies in the oral cavity have included bullets, impression materials, teeth, fish bones, needles, plastics, pistachio nuts, earrings, and the traumatic implantation of a toothbrush and various food products. ${ }^{1}$ These foreign bodies have been found by, inspection, palpation or as incidental findings on radiographs. A thorough history may establish an etiology and timeframe in which the foreign body was embedded in soft-tissue. Foreign body implantation has been reported in the literature from long but exact nature of the specimen is not always revealed. This article documents a case of a foreign body which was a guava seed embedded in buccal mucosa for 6 months which was ruled out by its typical radiographic presentation.
\end{abstract}

Keywords: Foreign bodies, Traumatic implantation, Radio visual graphy.

How to cite this article: Sharma S, Bumb D, Sen S, Priyadardshini S. An Unusual Case of Guava Seed Embedded in Buccal Mucosa for Past 6 Months. World J Dent 2014; 5(3):193-195.

Source of support: Nil

Conflict of interest: None

\section{CASE REPORT}

A 55-year-old male patient visited our out patient department with a chief complaint of swelling in right cheek since 5 to 6 months and mobile teeth in upper right back tooth region since 3 to 4 months and bleeding gums since 3 to 4 months. Swelling was of same size and did not increase or decrease in size. It was painless and no history of trauma was given by the patient. Patient did not give any history of difficulty in swallowing or dry mouth. There was no contributory medical and family history. Patient used to smoke bidi 10 per day since, 30 years and hookah 20 times/day since 30 years. On examining extraorally, there was no abnormality detected. Intraoral examination revealed marginal and attached gingiva to be reddish pink with blackish pigmentation. There was generalized recession and bleeding on probing present in all teeth (Fig. 1). Swelling was present on right buccal mucosa around 15 and 16 region above the occlusal plane (Fig. 2).

\footnotetext{
${ }^{1-4}$ Postgraduate Student

${ }^{1-4}$ Department of Oral Medicine and Radiology, Jaipur Dental College, Jaipur, Rajasthan, India

Corresponding Author: Shruti Sharma, Postgraduate Student Department of Oral Medicine and Radiology, Jaipur Dental College Jaipur, Rajasthan, India, e-mail: shruti19 sharma87@gmail.com
}

There was generalized mobility and furcation involvement (grade 3) in 16,17. On examination, the swelling was solitary, pink in color same as that of adjacent mucosa, shaped round, $1 \times 1 \mathrm{~cm}$ well-defined, firm to hard, tender on palpation and freely movable below the buccal mucosa (Fig. 3).

Based on the above data, differential diagnosis of minor salivary gland sialolith, phelobolith, cysticercosis was considered. Smoker's melanosis, chronic generalized periodontitis was made as a provisional diagnosis. Radio visual graphy (RVG) of right buccal mucosa with relation to swelling and exicisional biopsy was advised (Fig. 4). Patient's written consent was taken before performing biopsy. Radio visual graphy showed oval shaped structure with well-defined radiopaque borders along with less radiopaque internal structure. Because of this typical radiographic

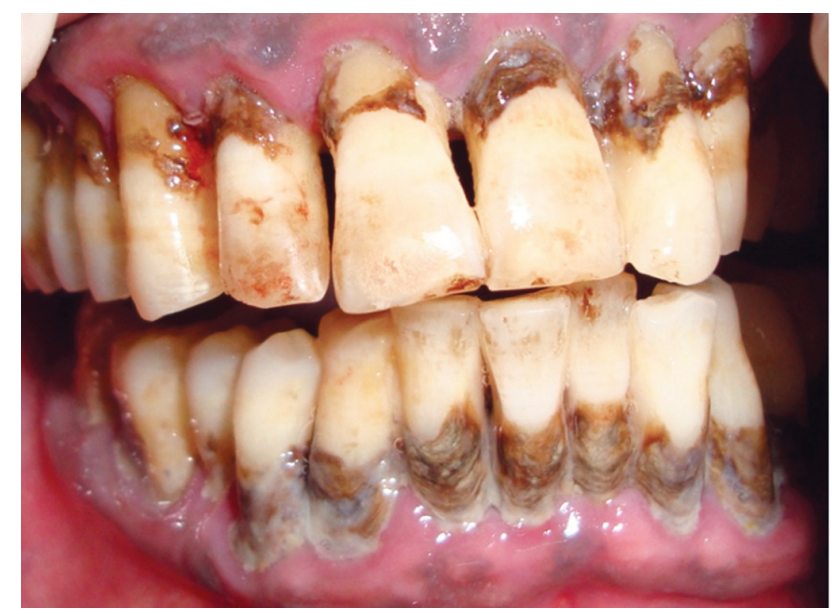

Fig. 1: Intraoral examination

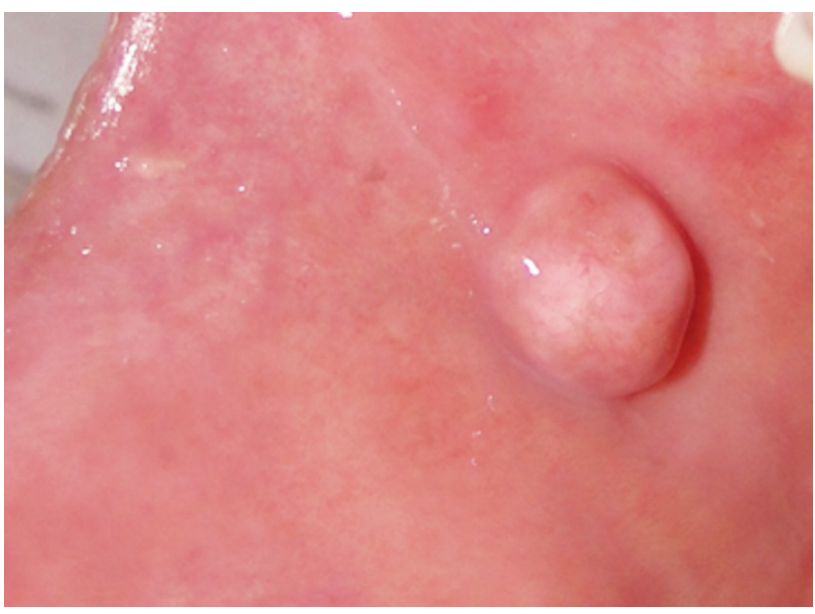

Fig. 2: Swelling on right buccal mucosa 


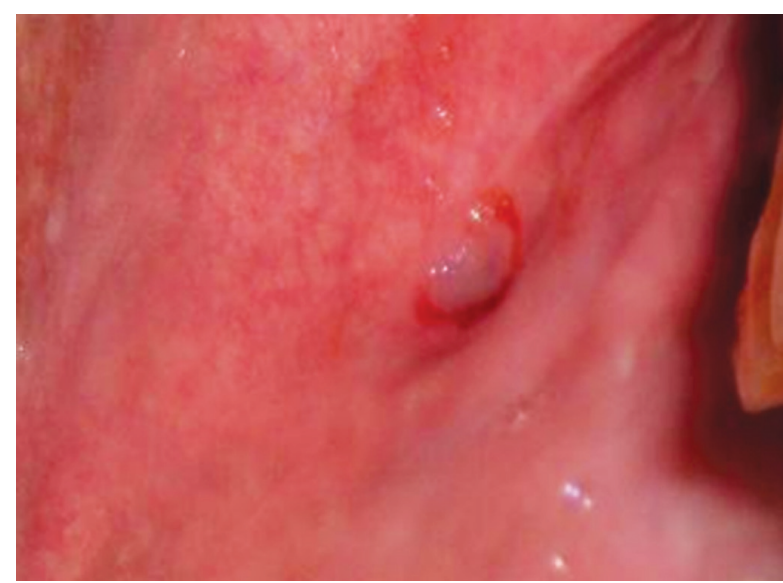

Fig. 3: Postoperative buccal mucosa

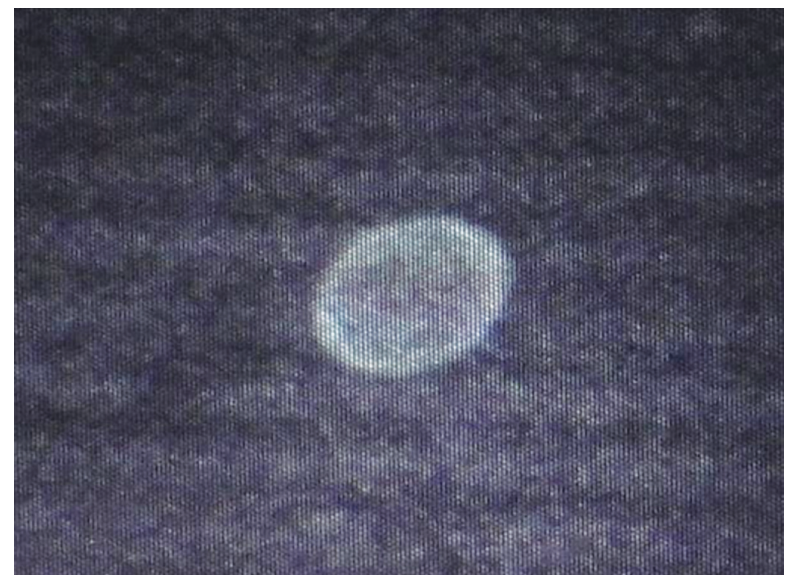

Fig. 4: RVG taken of right buccal mucosa

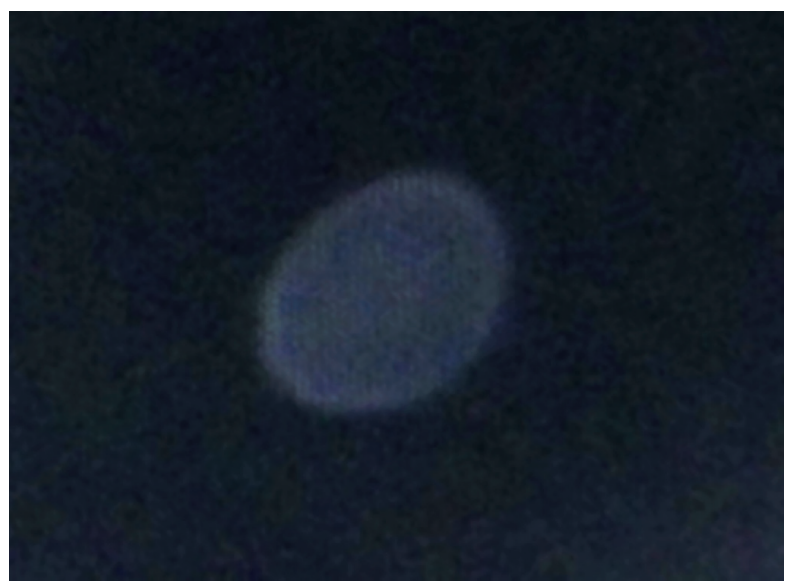

Fig. 5: RVG taken of the obtained specimen

presentation, all the entities were ruled out. Performing the excisional biopsy $5 \mathrm{~mm}$ oval shaped foreign body was taken out which had the same shape as seen in RVG (Fig. 5). This foreign particle was then sent to agriculture department, Jaipur for its evaluation. After inspecting the specimen and keeping in mind the size, shape, color and other significant features final diagnosis of foreign body (guava seed) embedded in buccal mucosa was made (Fig. 6).

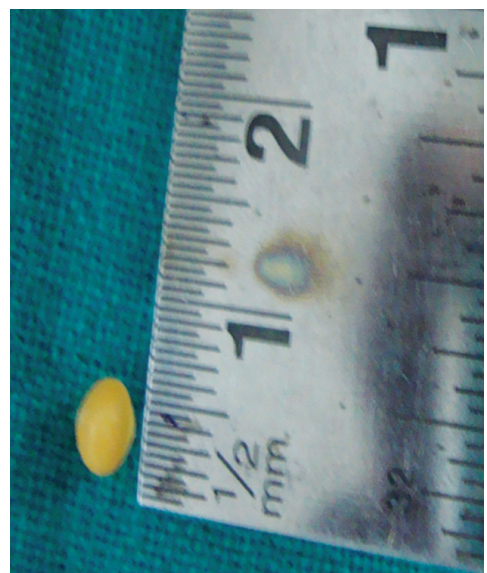

Fig. 6: Specimen obtained after biopsy

\section{DISCUSSION}

Foreign bodies and tissue reactions to foreign materials are commonly encountered in the oral cavity where $67 \%$ of such patients are asymptomatic and are accidental findings in routine dental procedures whereas $38 \%$ are symtomatic. ${ }^{2}$ Generally, four categories of patients are affected children mentally handicapped or mentally retarded persons adults with unusual sexual behavior, normal adults or children with predisposing factors or injurious situational problems. ${ }^{3}$ There are various modes of entrance, such as ingestion, insertion into a body cavity, or deposition into the body by a traumatic or iatrogenic injury. Various reasons can be motor vehicle accidents, assaults, and bullet wounds are common causes of traumatic foreign body's, apical deposition of endodontic materials, mucosal amalgam and graphite tattoos, traumatically introduced dental materials and instruments certain dietary habits particles in fish or other sea foods and legumes and pulses. ${ }^{1}$ They can be diagnosed by taking history of trauma, special dietary habits, oral habits, operation any other cosmetic procedure confirming it by radiograph. ${ }^{4}$ Radiographically, metal and glass appears dense radiopaque. Organic materials like wood are radiolucent whereas food and other particles show nonuniform radiopacity and radiolucency with ill defined margins. ${ }^{5}$ However, this case presents a different radiographic presentation. Keeping in mind all the morphological and radiographic features of the specimen, it was differentiated from other food products like pulses and corns. Taking the complete dietary history corns and other pulses were given as differential diagnosis for that specimen. However by confirming it with professor working with organic food firm, it was summarized that corn has a flat surface on one side which differs from the oval shape of the specimen and pulses generally are not as hard in density as of the specimen. Therefore, conclusion was drawn that careful inspection of the lesion, palpation, biopsy, radiographs and further evaluation of the specimen helped us to identify the exact nature of the specimen. 


\section{REFERENCES}

1. Vanessa, Ávila, Silveira S, et al. Intraosseous foreign-body granuloma in the buccal mucosa subsequent to a 20 -year-old work-related accident. Med Oral Patol Oral Cir Bucal 2008 Oct 1;13(10):E657-E660.

2. Hodges ED, Allen KA, Durham T. Nail-biting and foreign body embedment: a review and case report. Pediatr Dent 1994 MayJune;16(3):236-238.
3. Kazikdas C, et al. An unusual foreign body in the buccal mucosa presenting as a gradually growing mass. Eur Arch Otorhinolaryngol 2005;262:875-877.

4. Philipsen HP, Peter A. Reichart pulse or hyaline ring granuloma. Review of the literature on etiopathogenesis of oral and extraoral lesions. Clin Oral Invest 2010;14:121-128.

5. Mac Leod S. Traumatic implantation of a toothbrush: an unusual hazard of oral hygiene. ASDCJ Dent Child 1989;13:69-70. 